TRANSACTIONS OF THE

AMERICAN MATHEMATICAL SOCIETY

Volume 356, Number 5, Pages 1957-1970

S 0002-9947(03)03369-5

Article electronically published on October 28, 2003

\title{
SEMILATTICES OF FINITELY GENERATED IDEALS OF EXCHANGE RINGS WITH FINITE STABLE RANK
}

\author{
F. WEHRUNG
}

\begin{abstract}
We find a distributive $(\vee, 0,1)$-semilattice $S_{\omega_{1}}$ of size $\aleph_{1}$ that is not isomorphic to the maximal semilattice quotient of any Riesz monoid endowed with an order-unit of finite stable rank. We thus obtain solutions to various open problems in ring theory and in lattice theory. In particular:

- There is no exchange ring (thus, no von Neumann regular ring and no $\mathrm{C}^{*}$-algebra of real rank zero) with finite stable rank whose semilattice of finitely generated, idempotent-generated two-sided ideals is isomorphic to $S_{\omega_{1}}$.

- There is no locally finite, modular lattice whose semilattice of finitely generated congruences is isomorphic to $S_{\omega_{1}}$.

These results are established by constructing an infinitary statement, denoted here by $\mathrm{URP}_{\mathrm{sr}}$, that holds in the maximal semilattice quotient of every Riesz monoid endowed with an order-unit of finite stable rank, but not in the semilattice $S_{\omega_{1}}$.
\end{abstract}

\section{INTRODUCTION}

The present work originates in the representation problem of distributive $(\vee, 0)$ semilattices as semilattices of compact (i.e., finitely generated) ideals of von Neumann regular rings; see Section 13 in the chapter "Recent Developments" in [8], or the survey paper 11. It is known that the original form of this problem has a negative solution, obtained by the author in 21, 23]: There exists a distributive $(\vee, 0)$-semilattice that is not isomorphic to the semilattice of all compact ideals of any von Neumann regular ring. However, the example thus obtained has size $\aleph_{2}$. This cardinality bound turns out to be optimal. More specifically:

- The main result in [24] states that every distributive $(\vee, 0)$-semilattice of size at most $\aleph_{1}$ is isomorphic to the semilattice of all compact ideals of some von Neumann regular (but not locally matricial) algebra.

- By a well-known unpublished note by G. M. Bergman 4], every countable distributive $(\mathrm{V}, 0)$-semilattice is isomorphic to the semilattice of all compact ideals of some locally matricial (thus unit-regular) algebra. Two other proofs of this result are presented in [11, one of them using the following equivalent form: Every countable distributive $(\vee, 0)$-semilattice is isomorphic to the maximal semilattice quotient $\nabla\left(G^{+}\right)$of the positive cone $G^{+}$of some dimension group $G$.

Received by the editors January 3, 2003 and, in revised form, April 2, 2003.

2000 Mathematics Subject Classification. Primary 06A12, 20M14, 06B10; Secondary 19K14.

Key words and phrases. Semilattice, distributive, monoid, refinement, ideal, stable rank, strongly separative, exchange ring, lattice, congruence. 
Although the cardinality gap seems, at first glance, to be filled by the above works, the stronger representability result obtained by Bergman (locally matricial algebras are very special sorts of regular rings) leads to the question of whether similar results hold for distributive semilattices of cardinality $\aleph_{1}$.

It turns out that this problem has already been considered by a number of researchers.

The $\aleph_{1}$ nabla problem. Is every distributive $(\vee, 0)$-semilattice of size $\aleph_{1}$ isomorphic to the semilattice of all compact ideals of some locally matricial ring?

To our knowledge, this problem was first published as Problem 10.1 in 11. It is also equivalent to Problem 3 in the list of twelve open problems concluding the survey paper 20 .

A very important step towards a solution of the $\aleph_{1}$ nabla problem is obtained by P. Růžička in [18, where it is proved, via an ingenious construction, that there exists a distributive $(\mathrm{V}, 0)$-semilattice that is not isomorphic to the maximal semilattice quotient of the positive cone of any dimension group. In size $\aleph_{1}$, the bridge between locally matricial rings and dimension groups is obtained by results in 10. However, P. Růžička's counterexample has size $\aleph_{2}$, thus it does not imply a priori a negative solution to the $\aleph_{1}$ nabla problem.

In the present paper, we obtain a full negative solution to the $\aleph_{1}$ nabla problem; see Theorems 4.3 and 5.1. Our counterexample $S_{\omega_{1}}$, introduced at the beginning of Section [5] is very easy to describe. The idea underlying its proof is a combination of the ideas of [19, Example 11.1] and [18, Section 3], and it is based on the construction of a certain infinitary semilattice-theoretical sentence $\mathrm{URP}_{\mathrm{sr}}$, a so-called uniform refinement property, that we present in Section 4. This property $\mathrm{URP}_{\mathrm{sr}}$ holds for the maximal semilattice quotient $\nabla(M)$ of any Riesz monoid $M$ endowed with an order-unit of finite stable rank (Theorem 4.3). On the other hand, it is a near triviality to verify that $S_{\omega_{1}}$ does not satisfy URP $\mathrm{Ur}_{\mathrm{sr}}$ (Theorem 5.1); hence $S_{\omega_{1}}$ is a counterexample to the $\aleph_{1}$ nabla problem. The negative property established for this example is stronger than the one considered in [18, both from set-theoretical (cardinality) and algebraic (strength of the assumptions on $M$ ) viewpoints.

In Section [6] we describe some consequences of Theorems 4.3 and 5.1 The semilattice counterexample $S_{\omega_{1}}$ of Section 5 is not isomorphic to the semilattice of all compact ideals of any von Neumann regular ring with finite stable rank (Corollary 6.1), in particular, $S_{\omega_{1}}$ is not isomorphic to the semilattice of all compact ideals of any unit-regular ring. An extension of this result to exchange rings (a more general class including von Neumann regular rings and $\mathrm{C}^{*}$-algebras of real rank zero) is proposed in Corollary 6.2 Furthermore, $S_{\omega_{1}}$ is not isomorphic to the semilattice of all compact congruences of any modular lattice which is locally finite, or, more generally, of locally finite length (Corollary 6.3). We also prove that $S_{\omega_{1}}$ is not isomorphic to the semilattice of all compact congruences of any locally finite "lower bounded" lattice (Proposition 6.5), however, we also prove an apparently much stronger statement in Corollary 6.7

In Section [7 we prove a few results, either positive or negative, about the preservation of $U_{R P}$ sr and related properties under direct limits of countable sequences.

We conclude the paper in Section 8 , with a few open problems. 


\section{BASIC CONCEPTS}

We mainly follow the notation and terminology in K. R. Goodearl's monograph [9]. For elements $a, b_{1}, \ldots, b_{n}$ in some partially ordered set $P$, let $a \leq b_{1}, \ldots, b_{n}$ abbreviate the conjunction of the statements $a \leq b_{i}$, for $1 \leq i \leq n$. We put

$$
\downarrow X=\{p \in P \mid \exists x \in X \text { such that } p \leq x\} \text {, for all } X \subseteq P,
$$

and we say that $X$ is cofinal in $P$, if $\downarrow X=P$. We also put $\downarrow p=\downarrow\{p\}$, for all $p \in P$. Let $\uparrow X$, being 'coinitial', and $\uparrow p$ be defined dually.

For a partially ordered abelian group $G$, we denote by $G^{+}$the positive cone of $G$. We put $\mathbb{N}=\mathbb{Z}^{+} \backslash\{0\}$. We denote by $\omega$ (resp., $\omega_{1}$ ) the first infinite (resp., uncountable) ordinal. Following the usual set-theoretical convention, every ordinal $\alpha$ is the set of all ordinals less than $\alpha$. For example, $\omega \backslash n=\{k<\omega \mid n \leq k\}$, for all $n<\omega$.

All our monoids will be denoted additively. Every commutative monoid $(M,+, 0)$ will be endowed with its algebraic quasi-ordering, defined by $x \leq y$ iff there exists $z \in M$ such that $x+z=y$. We define binary relations $\propto$ and $\asymp$ on $M$ by

$$
\begin{aligned}
& a \propto b \Longleftrightarrow \exists n \in \mathbb{N} \text { such that } a \leq n b, \\
& a \asymp b \Longleftrightarrow a \propto b \text { and } b \propto a,
\end{aligned}
$$

for all $a, b \in M$. Thus $\asymp$ is a monoid congruence of $M$ and the quotient $\nabla(M)=$ $M / \asymp$ is a semilattice, usually called the maximal semilattice quotient of $M$. We denote by $[a]$ the $\asymp$-equivalence class of $a$, for all $a \in M$, and we call the map $M \rightarrow \nabla(M), a \mapsto[a]$ the canonical projection from $M$ onto $\nabla(M)$; see also [11, 18].

An ideal of $M$ is a subset $I$ of $M$ such that $0 \in I$ and $x+y \in I$ iff $x, y \in I$, for all $x, y \in M$. An element $e \in M$ is an order-unit of $M$, if $x \propto e$ holds for all $x \in M$.

We say that $M$ is cancellative, if $a+c=b+c$ implies that $a=b$, for all $a, b$, $c \in M$. We say that $M$ is strongly separative, if $a+b=2 b$ implies that $a=b$, for all $a, b \in M$. Of course, every cancellative commutative monoid is strongly separative.

We say that $M$ is a refinement monoid, if $a_{0}+a_{1}=b_{0}+b_{1}$ in $M$ implies the existence of $c_{i, j} \in M$, for $i, j<2$, such that $a_{i}=c_{i, 0}+c_{i, 1}$ and $b_{i}=c_{0, i}+c_{1, i}$, for all $i<2$. We say that $M$ is a Riesz monoid, if $c \leq a+b$ in $M$ implies that there are $a^{\prime} \leq a$ and $b^{\prime} \leq b$ in $M$ such that $c=a^{\prime}+b^{\prime}$. Every refinement monoid is a Riesz monoid. The converse does not hold; however, the two definitions are equivalent for $M$ a semilattice. We call a semilattice satisfying these equivalent conditions distributive; see [12].

Remark 2.1. Let $M$ be a commutative monoid, define an equivalence relation $\equiv$ on $M$ by $x \equiv y$ if $x \leq y \leq x$, for all $x, y \in M$. Then $\equiv$ is a monoid congruence and we call the quotient $M / \equiv$ the maximal antisymmetric quotient of $M$. A sophisticated counterexample by C. Moreira dos Santos (see [16]) shows that even for a strongly separative refinement monoid $M$, the maximal antisymmetric quotient $M / \equiv$ may not have refinement. On the other hand, it is obvious that if $M$ is a Riesz monoid, then so is $M / \equiv$. Observe that $\nabla(M) \cong \nabla(M / \equiv)$.

We say that a partially ordered abelian group $G$ is an interpolation group (see [9]), if its positive cone $G^{+}$is a refinement monoid. We say that $G$ is directed, if $G=$ $G^{+}+\left(-G^{+}\right)$, and unperforated, if $m x \geq 0$ implies that $x \geq 0$, for all $m \in \mathbb{N}$ and all $x \in G$. A dimension group is an unperforated, directed interpolation group; see also [6]. 
In order to conveniently formulate our next lemma, we shall introduce some notation.

Notation 2.2. For a commutative monoid $M$ and a family $\left(a_{i}\right)_{i \in I}$ of elements of $M$, we denote by $\left\langle a_{i} \mid i \in I\right\rangle^{[\wedge 2]}$ the set of all elements of $M$ of the form

$$
\sum\left(x_{p}\left|p \subseteq I^{\prime},\right| p \mid=2\right),
$$

for some finite subset $I^{\prime}$ of $I$ and with $x_{\{i, j\}} \leq a_{i}, a_{j}$, for all distinct $i, j \in I^{\prime}$. We also put $A^{[\wedge 2]}=\langle a \mid a \in A\rangle^{[\wedge 2]}$, for any $A \subseteq M$.

Lemma 2.3. Let $M$ be a Riesz monoid, let $n \in \mathbb{N}$, and let $a_{0}, \ldots, a_{n-1}, b \in M$. If $a_{i} \leq b$, for all $i<n$, then there exists $x \in\left\langle a_{i} \mid i<n\right\rangle^{[\wedge 2]}$ such that $\sum_{i<n} a_{i} \leq b+x$.

Proof. By induction on $n$. The case $n=1$ is trivial. For $n=2$, let $a_{0}, a_{1} \leq b$ in $M$. There exists $t \in M$ be such that $a_{1}+t=b$. By $a_{0} \leq a_{1}+t$ and since $M$ is a Riesz monoid, there exists $x \leq a_{0}, a_{1}$ such that $a_{0} \leq x+t$. Therefore, $a_{0}+a_{1} \leq$ $a_{1}+t+x=b+x$.

Suppose the result established for some $n$. Let $a_{0}, a_{1}, \ldots, a_{n}, b \in M$ such that $a_{i} \leq b$, for all $i \leq n$. Put $a=\sum_{i \leq n} a_{i}$ and $a^{\prime}=\sum_{i<n} a_{i}$. Also put $A_{k}=$ $\left\langle a_{i} \mid i<k\right\rangle^{[\wedge 2]}$, for all $k \leq n+1$. By the induction hypothesis, there exists $x \in A_{n}$ such that $a^{\prime} \leq b+x$. Since $a_{n} \leq b+x$ and $M$ is a Riesz monoid, there exists $y \leq a^{\prime}, a_{n}$ such that $a \leq b+x+y$. From $y \leq a^{\prime}=\sum_{i<n} a_{i}$ and the assumption that

$M$ is a Riesz monoid, it follows that there are $a_{0}^{\prime} \leq a_{0}, \ldots, a_{n-1}^{\prime} \leq a_{n-1}$ such that $y=\sum_{i<n} a_{i}^{\prime}$. From $y \leq a_{n}$, it follows that $a_{i}^{\prime} \leq a_{i}, a_{n}$, for all $i<n$. But $x \in A_{n}$, thus $x+y \in A_{n+1}$.

\section{Stable RANK In COMmUtative MONOIDS}

Let $M$ be a commutative monoid and let $k$ be a positive integer. An element $e \in M$ has stable rank at most $k$, if $k e+a=e+b$ implies that $a \leq b$, for all $a$, $b \in M$. Of course, this is equivalent to saying that $k e+a \leq e+b$ implies that $a \leq b$, for all $a, b \in M$. This definition has at its origin a purely ring-theoretical notion, the Bass stable rank of a ring; see P. Ara's survey paper [2]. In particular, an exchange ring $R$ has stable rank at most $k$ iff the isomorphism class of $R$ has stable rank at most $k$ in the commutative monoid $V(R)$ of isomorphism classes of finitely generated projective right $R$-modules; see [2] Theorem 2.2].

For an element $e$ of $M$, we denote by $\operatorname{sr}_{M}(e)$ the least positive integer $k$ such that $e$ has stable rank at most $k$ in $M$ if it exists, $\infty$ otherwise.

In any commutative monoid, $2 e+a=e+b$ implies that $2(e+a)=(e+a)+b$, hence, in the presence of strong separativity, $e+a=b$. In particular, we obtain the following result.

Proposition 3.1. For any commutative monoid $M$, the following statements hold:

(i) If $M$ is cancellative, then every element of $M$ has stable rank at most 1.

(ii) If $M$ is strongly separative, then every element of $M$ has stable rank at most 2 .

We put

$$
\operatorname{SR}(M)=\{(x, y) \in M \times M \mid \forall a, b \in M, x+a \leq y+b \Rightarrow a \leq b\} .
$$


Obviously, $\operatorname{SR}(M)$ is a submonoid of $M \times M$. Observe that $\operatorname{sr}_{M}(e) \leq k$ iff $(k e, e) \in$ $\mathrm{SR}(M)$. As an immediate consequence of these two simple facts, we observe the following.

Lemma 3.2. Let $e$ be an element of a commutative monoid $M$. Then $\operatorname{sr}_{M}($ ne) $\leq$ $\operatorname{sr}_{M}(e)$, for all $n \in \mathbb{N}$.

\section{A NEW UNIFORM REFINEMENT PROPERTY}

The negative solutions to other representation problems, either of dimension groups or distributive semilattices, introduced in [21, 23, were obtained as counterexamples to special infinitary statements called uniform refinement properties. The reader may consult 20] for a discussion about various such statements defined for semilattices.

Our new uniform refinement property is significantly different from all previously known ones. It is inspired by the proof of the counterexample in [19, Example 11.1]. In order to state it, we find it convenient to use Notation 2.2. We say that a partially ordered set $P$ is $\aleph_{0}$-downward directed, if every at most countable subset of $P$ lies above some element of $P$. We shall use the following straightforward lemma several times.

Lemma 4.1. Let $\left(P_{n}\right)_{n<\omega}$ be a sequence of subsets of a $\aleph_{0}$-downward directed partially ordered set $P$. If $\bigcup_{n<\omega} P_{n}=P$, then there exists $n<\omega$ such that $P_{n}$ is coinitial in $P$.

Now we turn to the most important definition of the paper.

Definition 4.2. Let $S$ be a join-semilattice. For an element $e \in S$, we introduce the following statements:

- $\mathrm{URP}_{\mathrm{sr}}(e)$ : for all subsets $A$ and $B$ of $S$ such that $A$ is uncountable, $B$ is $\aleph_{0}$-downward directed, and

$$
a \leq e \leq a \vee b \text { for all }(a, b) \in A \times B,
$$

there exists $a \in A^{[\wedge 2]}$ such that $e \leq a \vee b$ for all $b \in B$.

- $\mathrm{URP}_{\mathrm{sr}}^{+}(e)$ : for all $a_{0}, a_{1} \in S$ and all $\aleph_{0}$-downward directed $B \subseteq S$, if

$$
e \leq a_{i} \vee b \text { for all } i<2 \text { and all } b \in B \text {, }
$$

then there exists $a \leq a_{0}, a_{1}$ such that $e \leq a \vee b$ for all $b \in S$.

We say that $S$ satisfies $\mathrm{URP}_{\mathrm{sr}}$ (resp., $\mathrm{URP}_{\mathrm{sr}}^{+}$), if it satisfies $\operatorname{URP}_{\mathrm{sr}}(e)$ (resp., $\left.\operatorname{URP}_{\mathrm{sr}}^{+}(e)\right)$, for every $e \in S$.

Of course, $\mathrm{URP}_{\mathrm{sr}}^{+}$implies $\mathrm{URP}_{\mathrm{sr}}$.

Our main result is the following.

Theorem 4.3. Let $M$ be a Riesz monoid and let $e \in M$. If e has finite stable rank in $M$, then the maximal semilattice quotient $\nabla(M)$ satisfies $\operatorname{URP}_{\mathrm{sr}}([e])$.

Proof. Put $S=\nabla(M)$ and $\boldsymbol{e}=[e] \in S$. Let $\boldsymbol{A}$ and $\boldsymbol{B}$ be subsets of $S$ such that $\boldsymbol{A}$ is uncountable, $\boldsymbol{B}$ is $\aleph_{0}$-downward directed, and $\boldsymbol{a} \leq \boldsymbol{e} \leq \boldsymbol{a} \vee \boldsymbol{b}$ for all $(\boldsymbol{a}, \boldsymbol{b}) \in \boldsymbol{A} \times \boldsymbol{B}$. It is convenient to write these sets as

$$
\boldsymbol{A}=\left\{\boldsymbol{a}_{i} \mid i \in I\right\} \text { and } \boldsymbol{B}=\left\{\boldsymbol{b}_{j} \mid j \in J\right\},
$$

where $i \mapsto \boldsymbol{a}_{i}$ is one-to-one, $J$ is a $\aleph_{0}$-downward directed partially ordered set, and $j \mapsto \boldsymbol{b}_{j}$ is order-preserving. Pick $a_{i} \in \boldsymbol{a}_{i}$ for all $i \in I$ and $b_{j} \in \boldsymbol{b}_{j}$ for all $j \in J$. For 
all $i \in I$, there exists $m_{i} \in I$ such that $a_{i} \leq m_{i} e$. Since $I$ is uncountable, there are $m \in \mathbb{N}$ and an uncountable subset $U$ of $I$ such that $m_{i}=m$ for all $i \in U$. Thus $a_{i} \leq m e$ for all $i \in U$. But $m e$ has finite stable rank in $M$ (see Lemma [3.2), thus we may replace $e$ by $m e$, so that we obtain

$$
a_{i} \leq e, \text { for all } i \in U \text {. }
$$

For all $(i, j) \in I \times J$, since $\boldsymbol{e} \leq \boldsymbol{a}_{i} \vee \boldsymbol{b}_{j}$, there exists $n_{i, j} \in \mathbb{N}$ such that $e \leq n_{i, j}\left(a_{i}+b_{j}\right)$. Put $J_{i, n}=\left\{j \in J \mid n_{i, j}=n\right\}$, for all $(i, n) \in I \times \mathbb{N}$. For all $i \in I$, the equality $J=\bigcup_{n \in \mathbb{N}} J_{i, n}$ holds. Thus, by Lemma 4.1, there exists $n_{i} \in \mathbb{N}$ such that $J_{i, n_{i}}$ is coinitial in $J$. Since $U$ is uncountable, there are $n \in \mathbb{N}$ and an uncountable subset $V$ of $U$ such that $n_{i}=n$ for all $i \in V$. Observe that, by construction,

$$
e \leq n\left(a_{i}+b_{j}\right) \text {, for all } i \in V \text { and } j \in J_{i, n} .
$$

Put $k=\operatorname{sr}_{M}(e)$. We pick a subset $X \subset V$ with exactly $n k+1$ elements. By (4.1), the fact that $X$ is a subset of $U$, and Lemma 2.3, it follows that

$$
\sum_{i \in X} a_{i} \leq e+a, \text { for some } a \in\left\langle a_{i} \mid i \in X\right\rangle^{[\wedge 2]} .
$$

Observe that the element $\boldsymbol{a}=[a]$ of $S$ belongs to $\left\langle\boldsymbol{a}_{i} \mid i \in X\right\rangle^{[\wedge 2]}$, thus (since $i \mapsto \boldsymbol{a}_{i}$ is one-to-one) to $\boldsymbol{A}^{[\wedge 2]}$. Let $j \in J$. For all $i \in I$, the subset $J_{i, n}=J_{i, n_{i}}$ is coinitial in $J$, thus there exists $\varphi(i) \in J_{i, n}$ such that $\varphi(i) \leq j$. It follows from (4.2) that $e \leq n\left(a_{i}+b_{\varphi(i)}\right)$. Therefore, adding together all those inequalities for $i \in X$ and using (4.3), we obtain that

$$
(n k+1) e \leq n \sum_{i \in X} a_{i}+n \sum_{i \in X} b_{\varphi(i)} \leq n e+n a+n \sum_{i \in X} b_{\varphi(i)},
$$

thus, since $\operatorname{sr}_{M}(n e) \leq k$ (see Lemma 3.2),

$$
e \leq n a+n \sum_{i \in X} b_{\varphi(i)} .
$$

By applying to this inequality the canonical projection from $M$ onto $\nabla(M)$ and by using the fact that $\boldsymbol{b}_{\varphi(i)} \leq \boldsymbol{b}_{j}$ for all $i \in X$, we obtain the inequalities

$$
\boldsymbol{e} \leq \boldsymbol{a} \vee \bigvee_{i \in X} \boldsymbol{b}_{\varphi(i)} \leq \boldsymbol{a} \vee \boldsymbol{b}_{j}
$$

Hence the element $\boldsymbol{a} \in S$ is as required.

Remark 4.4. Although the assumptions made in the statement of Theorem 4.3 are not the weakest possible, they are probably the weakest that can be stated conveniently while meeting an application range as wide as possible within mathematical practice. Nevertheless, since one can never be sure about future applications, we list here some possible weakenings of the assumptions of Theorem 4.3 that lead to the same conclusion.

- Refinement assumption: for all $m \in \mathbb{N}$ and all $a_{0}, \ldots, a_{m-1}, b \in M$, if $a_{i} \leq b$ for all $i<m$, then there are $n \in \mathbb{N}$ and $x \in\left\langle n a_{i} \mid i<m\right\rangle^{[\wedge 2]}$ such that $\sum_{i<m} a_{i} \leq b+x$.

- Stable rank assumption: for all $m \in \mathbb{N}$, there exists $k \in \mathbb{N}$ such that for all $a, b \in M$, if $k m e+a \leq m e+b$, then $a \propto b$.

Corollary 4.5. Let $M$ be a strongly separative Riesz monoid. Then $\nabla(M)$ satisfies $\mathrm{URP}_{\mathrm{sr}}$. 


\section{THE EXAMPLE}

We first recall some constructions used in [18]. For an infinite cardinal $\kappa$, we denote by $\mathcal{B}_{\kappa}$ the interval algebra of $\kappa$, that is, the Boolean subalgebra of the powerset of $\kappa$ generated by all intervals of $\kappa$. Then we put

$$
\begin{aligned}
& \mathcal{J}_{\kappa}=\left\{x \in \mathcal{B}_{\kappa} \mid \exists \alpha<\kappa \text { such that } x \subseteq \alpha\right\}, \\
& \mathcal{F}_{\kappa}=\left\{x \in \mathcal{B}_{\kappa} \mid \exists \alpha<\kappa \text { such that } \kappa \backslash \alpha \subseteq x\right\}, \\
& D_{\kappa}=\{x \subseteq \kappa \mid \text { either } x \text { is finite or } x=\kappa\} .
\end{aligned}
$$

Observe that $D_{\kappa}$ is a distributive lattice with zero. We again use the notation $D^{-}=D \backslash\{\varnothing\}$, and we put

$$
S_{\kappa}=\left(\{\varnothing\} \times \mathcal{J}_{\kappa}\right) \cup\left(D_{\kappa}^{-} \times \mathcal{F}_{\kappa}\right) \quad\left(\text { thus } S_{\kappa} \subseteq D_{\kappa} \times \mathcal{B}_{\kappa}\right),
$$

endowed with its componentwise ordering. This is a particular case of a construction introduced in [18]. Observe that $S_{\kappa}$ is not a lattice (the meet of two elements of $D_{\kappa}^{-}$may be empty), however, it is a distributive $(\vee, 0,1)$-semilattice (see [18, Lemma 3.3]). Observe also that $S_{\kappa}$ has size $\kappa$.

Theorem 5.1. The distributive $(\vee, 0)$-semilattice $S_{\omega_{1}}$ does not satisfy $\operatorname{URP}_{\mathrm{sr}}(e)$, where we put $e=\left(\omega_{1}, \omega_{1}\right)$, the largest element of $S_{\omega_{1}}$.

Proof. We put $A=\left\{a_{\xi} \mid \xi<\omega_{1}\right\}$ and $B=\left\{b_{\xi} \mid \xi<\omega_{1}\right\}$, where we put

$$
a_{\xi}=\left(\{\xi\}, \omega_{1}\right) \text { and } b_{\xi}=\left(\omega_{1}, \omega_{1} \backslash \xi\right) \text {, for all } \xi<\omega_{1} .
$$

It is obvious that $A$ is uncountable, $B$ is $\aleph_{0}$-downward directed, and $a \leq e \leq a \vee b$ (in fact, $e=a \vee b$ ) for all $(a, b) \in A \times B$. For any $a \in\left\langle a_{\xi} \mid \xi<\omega_{1}\right\rangle^{\lceil\wedge 2]}$, there exists an ordinal $\alpha<\omega_{1}$ such that $a \leq(\varnothing, \alpha)$. Hence $a \vee b_{\alpha+1} \leq\left(\omega_{1}, \omega_{1} \backslash\{\alpha\}\right)<e$, which completes the proof.

Thus, by using Theorem 4.3, we obtain the following.

Corollary 5.2. Let $M$ be a Riesz monoid in which there is an order-unit of finite stable rank. Then $S_{\omega_{1}}$ is not isomorphic to $\nabla(M)$.

Also, by using Corollary 4.5 we obtain the following.

Corollary 5.3. There exists no strongly separative refinement monoid $M$ such that $\nabla(M) \cong S_{\omega_{1}}$. In particular, there exists no interpolation group (thus, no dimension group) $G$ such that $\nabla\left(G^{+}\right) \cong S_{\omega_{1}}$.

\section{Consequences in Ring theory and lattice theory}

In this section we shall reap some consequences of Theorem 4.3 and Theorem 5.1 thus answering a few open questions in ring theory and lattice theory. Throughout this section, we shall denote by $S_{\omega_{1}}$ the semilattice constructed in Section 5 .

6.1. Ideal lattices of von Neumann regular rings. For a von Neumann regular ring $R$, the monoid $V(R)$ of isomorphism classes of finitely generated projective right $R$-modules is a refinement monoid (see [8, Chapter 2]); it is, in addition, conical, that is, it satisfies the quasi-identity $x+y=0 \Rightarrow x=y=0$. Furthermore, the lattice of (two-sided) ideals of $R$ is isomorphic to the lattice of ideals of $V(R)$; see [11] Proposition 7.3].

We say that $R$ is strongly separative, if the monoid $V(R)$ is strongly separative. This notion is the monoid-theoretical translation of a purely ring-theoretical notion; 
see [3]. We observe, for a given von Neumann regular ring $R$, that the following implications hold:

locally matricial $\Rightarrow$ unit-regular $\Rightarrow$ strongly separative $\Rightarrow$ finite stable rank.

Corollary 6.1. There is no von Neumann regular ring $R$ with finite stable rank such that $S_{\omega_{1}}$ is isomorphic to the semilattice of all compact ideals of $R$.

Corollary 6.1]implies immediately that $S_{\omega_{1}}$ is not isomorphic to the semilattice of all compact ideals of any strongly separative (resp., unit-regular, locally matricial) ring. By contrast, we recall that every countable distributive $(\mathrm{V}, 0)$-semilattice is isomorphic to the semilattice of all compact ideals of some countable dimensional locally matricial ring (over any given field); see [4, 11].

We can even formulate an extension of Corollary 6.1 to more general rings called exchange rings. Recall (see the survey paper [2]) that every von Neumann regular ring or every $C^{*}$-algebra with real rank zero is an exchange ring. For an arbitrary exchange ring $R$, the canonical map from the ideal lattice of $R$ to the ideal lattice of $V(R)$ is surjective, and the inverse image of a singleton $\{I\}$ is the interval $\left[J_{0}, J_{1}\right]$, where $J_{0}$ is the ideal generated by all idempotents $e \in R$ such that $[e R] \in I$, and $J_{1}$ is the intersection of all primitive ideals containing $J_{0}$ (see [17. Teorema 4.1.7]). In particular, the maximal semilattice quotient of $V(R)$ is isomorphic to the semilattice of all compact idempotent-generated ideals of $R$. Hence we obtain the following.

Corollary 6.2. There is no exchange ring $R$ with finite stable rank such that $S_{\omega_{1}}$ is isomorphic to the semilattice of all compact idempotent-generated ideals of $R$.

6.2. Congruence lattices of modular lattices. We say that a lattice $K$ has locally finite length, if every finitely generated sublattice of $K$ has finite length. In particular, all locally finite lattices and all direct limits of finite-dimensional projective geometries have locally finite length.

Corollary 6.3. There is no modular lattice $K$ of locally finite length such that the semilattice $\mathrm{Con}_{\mathrm{c}} K$ of all compact congruences of $K$ is isomorphic to $S_{\omega_{1}}$.

Proof. Suppose that $S_{\omega_{1}} \cong \operatorname{Con}_{\mathrm{c}} K$, for a modular lattice $K$ of locally finite length. It follows from [22. Corollary 2.3] that $S_{\omega_{1}}$ is isomorphic to $\nabla(M)$, for $M=\operatorname{Dim} K$, the so-called dimension monoid of $K$. However, the dimension monoid of a modular lattice of finite length is a finitely generated free commutative monoid (see [22. Proposition 5.5]), and the dimension monoid functor preserves direct limits (see 22, Proposition 1.4]). Hence, since $K$ is modular with locally finite length, the monoid Dim $K$ is a direct limit of free commutative monoids, and thus it is the positive cone of a dimension group $G$. Hence,

$$
S_{\omega_{1}} \cong \nabla(\operatorname{Dim} K)=\nabla\left(G^{+}\right),
$$

a contradiction by Corollary 5.3 ,

In contrast, we recall that every distributive $(\vee, 0)$-semilattice of size at most $\aleph_{1}$ is isomorphic to the semilattice of all compact congruences of some locally finite relatively complemented lattice with zero (see [13]), and also isomorphic to the semilattice of all compact ideals of some von Neumann regular algebra, and to the semilattice of all compact congruences of some sectionally complemented, modular (but not locally finite) lattice (see [24]). 
6.3. Congruence lattices of lower bounded lattices. For lattices $K$ and $L$, a lattice homomorphism $f: K \rightarrow L$ is lower bounded, if $\{x \in K \mid a \leq f(x)\}$ is either empty or has a least element, for every $a \in L$. As in [1], we say that a lattice $L$ is lower bounded, if every homomorphism from a finitely generated lattice to $L$ is lower bounded. There are many equivalent definitions of lower boundedness for finite lattices, the simplest of them being that $L$ has as many join-irreducible elements as its congruence lattice Con $L$; see [7, Lemma 2.40]. Every lower bounded lattice is join-semidistributive, that is, it satisfies the quasi-identity

$$
x \vee y=x \vee z \Longrightarrow x \vee y=x \vee(y \wedge z) \text {. }
$$

Both properties of lower boundedness and join-semidistributivity are antithetical to modularity, as, for example, every join-semidistributive modular lattice is distributive. This antithesis also explains why results proved for modular lattices are often worth investigating for join-semidistributive, or lower bounded, lattices.

Congruence lattices of finite lower bounded lattices are fully characterized in [14]. In particular, not every finite distributive lattice is isomorphic to the congruence lattice of some finite lower bounded lattice, the three-element chain $\mathbf{3}$ being the simplest such example. On the dimension side, the following result is a consequence of [25] Corollary 6.3].

Proposition 6.4. The dimension monoid $\operatorname{Dim} L$ of any finite lower bounded lattice $L$ is strongly separative.

Since the Dim functor preserves direct limits, the result of Proposition 6.4 extends immediately to locally finite lower bounded lattices. Hence, from the isomorphism $\nabla(\operatorname{Dim} L) \cong \operatorname{Con}_{\mathrm{c}} L$ and by Corollary 5.3 we immediately obtain the following.

Proposition 6.5. There is no locally finite, lower bounded lattice $L$ such that $\operatorname{Con}_{\mathrm{c}} L \cong S_{\omega_{1}}$.

On the other hand, a much more striking negative conclusion can be reached by totally different means, via the following result.

Proposition 6.6. Let $L$ be a lower bounded lattice. If $\operatorname{Con} L$ is finite, then so is $L$.

Proof. It is proved in [5] that every finite lower bounded lattice $L$ satisfies the following Day-Pudlák-Túma property (DPT):

$\left[\Theta\left(a^{\prime}, a\right)=\Theta\left(b^{\prime}, b\right)\right.$ and $a^{\prime}<a$ and $\left.b^{\prime}<b\right] \Rightarrow a \wedge b \not \leq a^{\prime}, b^{\prime}$, for all $a, a^{\prime}, b, b^{\prime} \in L$, where $\Theta(x, y)$ denotes the congruence of $L$ generated by the pair $(x, y)$. By [1. Theorem 2.1], this result can be extended to arbitrary lower bounded lattices. Now suppose that $\operatorname{Con} L$ is finite; put $n=|\operatorname{Con} L|-1$. If there exists a chain $x_{0}<x_{1}<\cdots<x_{n+1}$ in $L$, then there are $i<j$ such that $\Theta\left(x_{i}, x_{i+1}\right)=\Theta\left(x_{j}, x_{j+1}\right)$. Thus, by (DPT), $x_{i+1}=x_{i+1} \wedge x_{j+1} \not \leq x_{j}$, a contradiction. Hence $L$ has length at most $n$, but it is lower bounded, thus join-semidistributive; by a classical result of B. Jónsson and J. E. Kiefer (see [15]; also [7, Theorem 5.59]), $|L| \leq 2^{n}$.

The upper bound $|L| \leq 2^{n}$ is rather crude. Indeed, since $L$ is finite lower bounded, there exists (see [7, Lemma 2.40]) a bijection between the set $\mathrm{J}(L)$ of join-irreducible elements of $L$ and $\mathrm{J}(\operatorname{Con} L)$. In particular, $|L| \leq 2^{|\mathrm{J}(\operatorname{Con} L)|}$.

Corollary 6.7. There is no lower bounded lattice $L$ such that $\operatorname{Con} L \cong \mathbf{3}$. 
Proof. If Con $L \cong \mathbf{3}$, then, by Proposition 6.6, $L$ is finite. However, there is no such finite lower bounded lattice $L$; see [14.

Observe that no assumption of local finiteness is necessary in Proposition 6.6 and Corollary6.7 On the other hand, unlike $S_{\omega_{1}}$, the $(\vee, 0)$-semilattice $\mathbf{3}$ is the maximal semilattice quotient of a strongly separative refinement monoid $M$-in fact, by Bergman's Theorem, $M$ may be chosen as the positive cone of some dimension group. See the related Problem [2.

\section{Preservation under Countable direct limits}

The following easy result introduces another class of distributive semilattices with $\mathrm{URP}_{\mathrm{sr}}$, defined in a completely different way.

Proposition 7.1. Let $S$ be a distributive join-semilattice such that $\downarrow a_{0} \cap \downarrow a_{1}$ has an at most countable cofinal subset, for all $a_{0}, a_{1} \in S$. Then $S$ satisfies $\mathrm{URP}_{\mathrm{sr}}^{+}$.

Proof. Let $e, a_{0}, a_{1} \in S$ and let $B$ be an $\aleph_{0}$-downward directed subset of $S$ such that $e \leq a_{i} \vee b$ for all $i<2$ and all $b \in B$. By assumption, there exists an increasing cofinal sequence $\left\{a^{(n)} \mid n<\omega\right\}$ in $\downarrow a_{0} \cap \downarrow a_{1}$. Suppose that for all $n<\omega$, there exists $b_{n} \in B$ such that $e \not \leq a^{(n)} \vee b_{n}$. By assumption, there exists $b \in B$ such that $b \leq b_{n}$ for all $n<\omega$; hence $e \not a^{(n)} \vee b$ for all $n<\omega$. On the other hand, $e \leq a_{i} \vee b$ for all $i<2$, thus, since $S$ is distributive, $e \leq x \vee b$ for some $x \leq a_{0}, a_{1}$. Pick $n<\omega$ such that $x \leq a^{(n)}$; then $e \leq a^{(n)} \vee b$, a contradiction. So we have proved that there exists $n<\omega$ such that $e \leq a^{(n)} \vee b$ for all $b \in B$.

Observe the following immediate corollary of Proposition 7.1

Corollary 7.2. Every direct limit of a countable sequence of distributive lattices and join-homomorphisms satisfies $\mathrm{URP}_{\mathrm{sr}}^{+}$(thus also $\mathrm{URP}_{\mathrm{sr}}$ ).

Compare with [20 Proposition 2.11].

We shall soon see (Proposition 7.7) that the class of all semilattices satisfying $\mathrm{URP}_{\mathrm{sr}}$ is not closed under direct limits of countable sequences. However, the following related positive result holds.

Proposition 7.3. Let $\left(S_{n}\right)_{n<\omega}$ be an increasing sequence of join-subsemilattices of a join-semilattice $S$ such that $S=\bigcup_{n<\omega} S_{n}$. If all $S_{n}$-s satisfy $\mathrm{URP}_{\mathrm{sr}}$ (resp., $\left.\mathrm{URP}_{\mathrm{sr}}^{+}\right)$, then so does $S$.

Proof. We provide a proof for $\mathrm{URP}_{\mathrm{sr}}$; the proof for $\mathrm{URP}_{\mathrm{sr}}^{+}$is similar. Let $e \in S$ and let $A$ and $B$ be subsets of $S$, where $A$ is uncountable, $B$ is $\aleph_{0}$-downward directed, and $a \leq e \leq a \vee b$ for all $(a, b) \in A \times B$. By Lemma 4.1 there exists $n<\omega$ such that $B_{n}=B \cap S_{n}$ is coinitial in $B$-in particular, it is $\aleph_{0}$-downward directed; furthermore, we may assume that $A_{n}=A \cap S_{n}$ is uncountable. Since $a \leq e \leq a \vee b$ for all $(a, b) \in A_{n} \times B_{n}$ and $S_{n}$ satisfies $\mathrm{URP}_{\mathrm{sr}}$, there exists $a \in A_{n}{ }^{[\wedge 2]}$ such that $e \leq a \vee b$ for all $b \in B_{n}$. So $a \in A^{[\wedge 2]}$ and, since $B_{n}$ is coinitial in $B$, the inequality $e \leq a \vee b$ holds for all $b \in B$.

Denote by $\mathcal{C}$ the class of all distributive $(\vee, 0)$-semilattices without any decreasing $\omega_{1}$-chain. Observe that any nonempty $\aleph_{0}$-downward directed subset of any member $S$ of $\mathcal{C}$ has a least element. Hence the following result holds.

Proposition 7.4. Every member of $\mathfrak{C}$ satisfies $\mathrm{URP}_{\mathrm{sr}}^{+}$. 
Denote by $\mathcal{C}_{\omega}$ the class of all direct limits of countable sequences of members of $\mathcal{C}$, and by fin the Fréchet filter on $\omega$. Furthermore, for a set $X$ and a sequence $x=\left\langle x_{n} \mid n<\omega\right\rangle$ in $X^{\omega}$, we denote by $\left[x_{n} \mid n<\omega\right]$ the equivalence class of $x$ modulo fin in $X^{\omega}$.

Lemma 7.5. Let $S$ and $C$ be distributive $(\vee, 0)$-semilattices with $C \in \mathcal{C}$. If $S$ embeds into the reduced power $C^{\omega} /$ fin, then $S$ belongs to $\mathcal{C}_{\omega}$.

Proof. Let $f: S \hookrightarrow C^{\omega} /$ fin be a $(\vee, 0)$-embedding. Denote by $S^{\prime}$ its image. For all $n<\omega$, let $\pi_{n}: C^{\omega \backslash n} \rightarrow C^{\omega} /$ fin be the map defined by

$$
\pi_{n}\left(\left\langle x_{k} \mid n \leq k<\omega\right\rangle\right)=\left[x_{k} \mid k<\omega\right] \text {, where we put } x_{0}=\cdots=x_{n-1}=0 .
$$

It is not hard to verify that $S_{n}=\pi_{n}^{-1}\left[S^{\prime}\right]$ is a distributive $(\vee, 0)$-subsemilattice of $C^{\omega \backslash n}$. Put

$$
\rho_{n}=f^{-1} \circ \pi_{n} \Upsilon_{S_{n}} .
$$

Furthermore, for $m \leq n<\omega$, it is possible to define a $(\vee, 0)$-homomorphism $\rho_{m, n}: S_{m} \rightarrow S_{n}$ by the rule $\rho_{m, n}(x)=\left.x\right|_{\omega \backslash n}$, for all $x \in S_{m}$.

It is routine to verify that $S=\varliminf_{n<\omega} S_{n}$, with transition maps $\rho_{m, n}: S_{m} \rightarrow S_{n}$ and limiting maps $\rho_{n}: S_{n} \rightarrow S$. The semilattice $S_{n}$ is a distributive subsemilattice of $C^{\omega \backslash n}$, for all $n<\omega$, and $C \in \mathcal{C}$, thus $S_{n} \in \mathcal{C}$.

Now we shall present a construction which proves that the semilattice $S_{\omega_{1}}$ of Section 5 belongs to $\mathcal{C}_{\omega}$. For maps $f, g$ from $\omega$ to $\omega+1$, let $f<^{*} g$ be the following statement:

$$
f<^{*} g \Longleftrightarrow\{n<\omega \mid f(n) \geq g(n)\} \text { is finite. }
$$

It is well-known that there exists a $\omega_{1}$-sequence $\left(f_{\alpha}\right)_{\alpha<\omega_{1}}$ of maps from $\omega$ to $\omega$ such that $\alpha<\beta$ implies that $f_{\alpha}<^{*} f_{\beta}$, for all $\alpha, \beta<\omega_{1}$. Let $f_{\omega_{1}}: \omega \rightarrow \omega+1$ denote the constant function with value $\omega$. Observe that

$$
\alpha<\beta \Longrightarrow f_{\alpha}<^{*} f_{\beta}, \text { for all } \alpha, \beta \leq \omega_{1} .
$$

Every element $x \in \mathcal{B}_{\omega_{1}}$ has a unique normal form,

$$
x=\bigcup_{i<n}\left[\alpha_{i}, \beta_{i}\right), \text { where } n<\omega \text { and } \alpha_{0}<\beta_{0}<\cdots<\alpha_{n-1}<\beta_{n-1} \leq \omega_{1} .
$$

Observe that the $\left[\alpha_{i}, \beta_{i}\right)$-s are exactly the maximal subintervals of $x$. For $x \in \mathcal{B}_{\omega_{1}}$ written in normal form as in (7.2), we put

$$
g_{k}(x)=\bigcup_{i<n}\left[f_{\alpha_{i}}(k), f_{\beta_{i}}(k)\right), \text { for all } k<\omega,
$$

where, of course, an interval $[x, y)$ is empty if $x \geq y$. Observe that for all large enough $k$, the following inequalities hold:

$$
f_{\alpha_{0}}(k)<f_{\beta_{0}}(k)<\cdots<f_{\alpha_{n-1}}(k)<f_{\beta_{n-1}}(k) .
$$

Hence, for such values of $k, 7.3)$ is an expression of $g_{k}(x)$ in normal form.

For an element $x \in D_{\omega_{1}}$ and $k<\omega$, we define $u_{k}(x) \in D_{\omega}$ by

$$
u_{k}(x)= \begin{cases}\left\{f_{\alpha}(k) \mid \alpha \in x\right\}, & \text { if } x \text { is finite } \\ \omega, & \text { if } x=\omega_{1}\end{cases}
$$

Finally, we put

$$
h(x, y)=\left[\left(u_{k}(x), g_{k}(y)\right) \mid k<\omega\right], \text { for all }(x, y) \in S_{\omega_{1}} .
$$


We leave to the reader the easy but somehow tedious proof of the following lemma. The main reason why it works is that containments between elements $x$ and $y$ of $S_{\kappa}$, for an infinite cardinal $\kappa$, can be expressed by inequalities between the endpoints of the maximal subintervals of $x$ and $y$. But then, inequalities between ordinals in $\omega_{1}+1$ can be "projected on" $\omega+1$ by using (7.1).

Lemma 7.6. The map $h$ is a $(\vee, 0)$-embedding from $S_{\omega_{1}}$ into $\left(S_{\omega}\right)^{\omega} /$ fin.

Observe that Lemma 7.6 does not hold "trivially", in the following sense. Although it is easy to prove that all the maps $u_{k}$, for $k<\omega$, are join-homomorphisms, this is not the case for the $g_{k}$ 's. However, this seemingly irregular behavior disappears "at the limit", as $k$ goes to infinity.

By using Lemmas 7.5 and 7.6 we obtain the following.

Proposition 7.7. The semilattice $S_{\omega_{1}}$ belongs to $\mathfrak{C}_{\omega}$.

By Theorem [5.1, Proposition 7.4, and Proposition 7.7, the class of all semilattices satisfying $\mathrm{URP}_{\mathrm{sr}}$ is not closed under direct limits of countable sequences. Compare with Corollary 7.2 and Proposition 7.3 .

Remark 7.8. Let $h: S_{\omega_{1}} \hookrightarrow\left(S_{\omega}\right)^{\omega} /$ fin be the previously constructed $(\vee, 0)$-embedding. It follows from Lemma 7.5 that $S_{\omega_{1}}$ is the direct limit of the lattices $T_{n}=\pi_{n}^{-1} h\left[S_{\omega_{1}}\right]$, for $n<\omega$, and all $T_{n}$-s belong to $\mathcal{C}$. Furthermore, the map $\rho_{n}=h^{-1} \circ \pi_{n}: T_{n} \rightarrow S_{\omega_{1}}$ is a surjective homomorphism of a very special kind; namely,

$$
\rho_{0}(x) \leq \rho_{0}(y) \Longleftrightarrow \exists u \in S_{\omega}^{(\omega)} \text { such that } x \leq y \vee u, \text { for all } x, y \in T_{0},
$$

where we denote by $S_{\omega}^{(\omega)}$ the ideal of $\left(S_{\omega}\right)^{\omega}$ that consists of all sequences with finite support. Hence,

$$
S_{\omega_{1}} \cong T_{0} / S_{\omega}^{(\omega)}
$$

It follows from this that $T_{0}$ is not isomorphic to $\nabla\left(G^{+}\right)$, for any interpolation group $G$. Indeed, otherwise, by (7.4), $S_{\omega_{1}}$ would be isomorphic to $\nabla\left((G / I)^{+}\right)$, where $I$ is the ideal of $G$ generated by all elements of $G^{+}$such that $[x]$ belongs to $S_{\omega}^{(\omega)}$. However, it follows from Corollary 5.3 that this is not possible. So we have obtained the following negative result: There exists a distributive $(\vee, 0,1)$ semilattice without descending $\omega_{1}$-chains that cannot be isomorphic to $\nabla\left(G^{+}\right)$for an interpolation group $G$.

This implies, in turn, the following negative result: $\mathrm{URP}_{\mathrm{sr}}$ is not sufficient to characterize all distributive $(\vee, 0,1)$-semilattices of the form $\nabla\left(G^{+}\right)$for $G$ an interpolation group.

\section{OPEN PROBLEMS}

Our most intriguing open problem is related to the following known results:

- Every $(\vee, 0)$-semilattice of the form $\lim _{n<\omega} D_{n}$, with all $D_{n}$ 's being distributive lattices with zero and all transition maps being $(\vee, 0)$-homomorphisms, is isomorphic to the semilattice of all compact congruences of some relatively complemented lattice with zero (see [26]).

- Every distributive lattice with zero is isomorphic to $\nabla\left(G^{+}\right)$, for some dimension group $G$ (see [11]).

Is it possible to unify these results? We can, for example, ask the following. 
Problem 1. Let $S=\lim _{\longrightarrow}{ }_{n} D_{n}$, with all $D_{n}$ 's being distributive lattices with zero and all transition maps being $(\vee, 0)$-homomorphisms. Does there exist a dimension group $G$ such that $S \cong \nabla\left(G^{+}\right)$?

The uniform refinement property $\mathrm{URP}_{\mathrm{sr}}$ is of no help to solve Problem 1 negatively: indeed, by Corollary $7.2, S$ does satisfy $\mathrm{URP}_{\mathrm{sr}}$.

Problem 2. Let $M$ be a strongly separative refinement monoid. Does there exist an interpolation group $G$ such that $\nabla(M) \cong \nabla\left(G^{+}\right)$?

As in [18, we say that a partially ordered abelian group $G$ is weakly Archimedean, if for all $a, b \in G^{+}$, if $n a \leq b$ holds for all $n \in \mathbb{N}$, then $a=0$. By using one of the main results in [19, P. Růžička proves in [18] the following result: Every countable distributive $(\vee, 0)$-semilattice is isomorphic to $\nabla\left(G^{+}\right)$, for some weakly Archimedean dimension group $G$; see [18, Theorem 3.1].

We say that a partially ordered abelian group $G$ is Archimedean, if for all $a$, $b \in G$, if $n a \leq b$ for all $n \in \mathbb{N}$, then $a \leq 0$.

Problem 3. For a countable distributive $(\vee, 0)$-semilattice $S$, does there exist an Archimedean dimension group $G$ such that $\nabla\left(G^{+}\right) \cong S$ ?

Another problem, inspired by Bergman's Theorem, Corollary [6.3], and some open questions in [20], is the following.

Problem 4. For a countable distributive semilattice $S$, does there exist a modular lattice $K$, generating a locally finite variety, such that $\operatorname{Con}_{\mathrm{c}} K \cong S$ ?

The statement that $K$ generates a locally finite variety is stronger than the mere local finiteness of $K$. It is equivalent to saying that for every $n \in \mathbb{N}$, the cardinalities of all $n$-generated sublattices of $K$ are bounded by a positive integer.

Our next problem asks about lifting not semilattices, but diagrams of semilattices. We say that a diagram of semilattices, indexed by a partially ordered set $I$, is finite (resp., countable, dismantlable), if $I$ is finite (resp., countable, dismantlable). It is proved in 19 that every finite dismantlable diagram of finite Boolean semilattices can be lifted, with respect to the $\nabla$ functor, by a diagram of (positive cones of) dimension groups.

Problem 5. Can every countable dismantlable diagram of countable distributive $(\vee, 0)$-semilattices be lifted, with respect to the $\nabla$ functor, by a diagram of (positive cones of) dimension groups?

\section{ACKNOWLEDGMENT}

I thank the anonymous referee for his thoughtful report, which brought additional life and openness to the topics discussed in the paper.

\section{REFERENCES}

1. K. V. Adaricheva and V.A. Gorbunov, On lower bounded lattices, Algebra Universalis 46 (2001), 203-213. MR 2002c:06004

2. P. Ara, Stability properties of exchange rings, International Symposium on Ring Theory (Kyongju, 1999), Trends Math., Birkhäuser Verlag, Boston, MA, 2001, 23-42. MR 2002g:16012

3. P. Ara, K. R. Goodearl, K. C. O'Meara, and E. Pardo, Separative cancellation for projective modules over exchange rings, Israel J. Math. 105 (1998), 105-137. MR 99g:16006

4. G. M. Bergman, Von Neumann regular rings with tailor-made ideal lattices, Unpublished note (26 October 1986). 
5. A. Day, Characterization of finite lattices that are bounded homomorphic images of sublattices of free lattices, Canad. J. Math. 31 (1979), 69-78. MR 81h:06004

6. E. G. Effros, D. E. Handelman, and C.-L. Shen, Dimension groups and their affine representations, Amer. J. Math. 102 (1980), no. 2, 385-407. MR 83g:46061

7. R. Freese, J. Ježek, and J. B. Nation, "Free Lattices", Mathematical Surveys and Monographs 42, Amer. Math. Soc., Providence, 1995. viii+293 pp. MR 96c:06013

8. K. R. Goodearl, "Von Neumann Regular Rings", Pitman, London 1979; Second Ed. Krieger, Malabar, Fl., 1991. MR 93m:16006

9. _ "Partially Ordered Abelian Groups with Interpolation", Math. Surveys and Monographs 20, Amer. Math. Soc., Providence, 1986. MR 88f:06013

10. K. R. Goodearl and D. E. Handelman, Tensor products of dimension groups and $K_{0}$ of unitregular rings, Canad. J. Math. 38, no. 3 (1986), 633-658. MR 87i:16043

11. K. R. Goodearl and F. Wehrung, Representations of distributive semilattices in ideal lattices of various algebraic structures, Algebra Universalis 45 (2001), 71-102. MR 2002g:06008

12. G. Grätzer, "General Lattice Theory. Second edition", new appendices by the author with B. A. Davey, R. Freese, B. Ganter, M. Greferath, P. Jipsen, H. A. Priestley, H. Rose, E. T. Schmidt, S. E. Schmidt, F. Wehrung, and R. Wille, Birkhäuser Verlag, Basel, 1998. xx+663 p. MR 2000b:06001

13. G. Grätzer, H. Lakser, and F. Wehrung, Congruence amalgamation of lattices, Acta Sci. Math. (Szeged) 66 (2000), 339-358. MR 2001f:06010

14. G. Grätzer and F. Wehrung, On the number of join-irreducibles in a congruence representation of a finite distributive lattice, Algebra Universalis 49 (2003), 165-178.

15. B. Jónsson and J.E. Kiefer, Finite sublattices of a free lattice, Canad. J. Math. 14 (1962), 487-497. MR 25:1117

16. C. Moreira dos Santos, A refinement monoid whose maximal antisymmetric quotient is not a refinement monoid, Semigroup Forum 65, no. 2 (2002), 249-263. MR 2003c:20074

17. E. Pardo, Monoides de refinament $i$ anells d'intercanvi, Ph.D. Thesis, Universitat Autònoma de Barcelona, 1995.

18. P. Růžička, A distributive semilattice not isomorphic to the maximal semilattice quotient of the positive cone of any dimension group, J. Algebra 268, no. 1 (2003), 290-300.

19. J. Tüma and F. Wehrung, Liftings of diagrams of semilattices by diagrams of dimension groups, Proc. London Math. Soc. 87, no. 3 (2003), 1-28.

20. - A survey of recent results on congruence lattices of lattices, Algebra Universalis 48, no. 4 (2002), 439-471.

21. F. Wehrung, Non-measurability properties of interpolation vector spaces, Israel J. Math. 103 (1998), 177-206. MR 99g:06023

22. The dimension monoid of a lattice, Algebra Universalis 40, no. 3 (1998), 247-411. MR 2000i:06014

23. _ A uniform refinement property for congruence lattices, Proc. Amer. Math. Soc. 127, no. 2 (1999), 363-370. MR 99c:06007

24. - Representation of algebraic distributive lattices with $\aleph_{1}$ compact elements as ideal lattices of regular rings, Publ. Mat. (Barcelona) 44, no. 2 (2000), 419-435. MR 2001k:16015

25. _ From join-irreducibles to dimension theory for lattices with chain conditions, J. Algebra Appl. 1, no. 2 (2002), 215-242. MR 2003f:06007

26. __ Forcing extensions of partial lattices, J. Algebra 262, no. 1 (2003), 127-193.

Département de Mathématiques, CNRs, UmR 6139, Université de Caen, Campus it, B.P. 5186, 14032 Caen Cedex, France

E-mail address: wehrung@math.unicaen.fr

$U R L:$ http://www.math. unicaen.fr/ ${ }^{\sim}$ wehrung 